\title{
Alain Badiou : un philosophe face au concept de modèle
}

\author{
Franck Varenne \\ Philosophe, Université de Rouen, UFR LSH, Département de philosophie, 76821 Mont-Saint-Aignan, France
}

\author{
Mots-clés : \\ Badiou ; \\ modèle ; \\ matérialisme ; \\ pragmatisme; \\ pluralisme
}

\begin{abstract}
Résumé - En 1969, le philosophe français Alain Badiou écrivait un petit ouvrage intitulé Le concept de modèle - Introduction à une épistémologie matérialiste des mathématiques. Une récente réimpression donne l'opportunité de revenir sur cet ouvrage et d'analyser ses arguments d'abord pour eux-mêmes. Ce texte vise donc essentiellement à présenter et à expliquer les arguments de Badiou contre la vision représentationaliste des modèles dans les sciences empiriques et pour l'interprétation matérialiste des systèmes formels couplés aux modèles sémantiques en mathématiques. À l'heure où les pratiques de modélisation et de simulation se développent encore davantage, et ce, dans toutes les disciplines, comme aussi à leurs frontières mutuelles, il est stimulant de relire une telle critique de l'illusoire pouvoir représentationnel des modèles et des simulations. Cependant, la question est posée de savoir si une telle critique est toujours valable contre les actuels systèmes de modélisation et de simulation complexes. Pour finir, il est suggéré qu'une telle épistémologie ne rejette pas tant une sorte d'empirisme qu'une sorte de pluralisme. Ce pourrait être une des explications du fait que les épistémologues des modèles ont de nos jours davantage été conduits à réactualiser les analyses du pragmatisme que celles du praxisme (ou théorie de la praxis) marxiste.
\end{abstract}

\section{Keywords:}

Badiou;

model;

materialism;

pragmatism;

pluralism

\begin{abstract}
Alain Badiou: a philosopher facing the concept of model. In 1969, the influent French philosopher Alain Badiou published a book called The concept of Model: An Introduction to the Materialist Epistemology of Mathematics. A recent reprint gives the opportunity to trace back and analyze its main arguments. This paper essentially aims to present and explain Badiou's arguments against the representationalist vision of models in empirical sciences and for a materialist interpretation of formal systems coupled with semantic models in mathematics. Now that the practices of scientific modeling and simulation have extensively spread across all scientific disciplines and their mutual frontiers, it is stimulating to revisit such a critic of the misleading representational power of models and simulations. However, the question is asked whether such a critique is still relevant against current complex modeling and simulation systems. Finally, it is suggested that what is rejected in such an epistemology is some kind of pluralism. This strict rejection can be one of the explaining factors that have led most current epistemologists of models to regenerate pragmatist analyses instead of the Marxist "praxist" ones.
\end{abstract}

\section{Introduction}

«C'est parce qu'il est vraiment de son temps - les années soixante - que ce petit livre peut être du nôtre. » L'avantdernière phrase de la préface qu'Alain Badiou vient d'écrire à l'occasion de la réédition de Le concept de modèle soutient une thèse forte et provocante, celle de l'actualité persistante de cet ouvrage critique d'abord paru en 1969.

Par là, son auteur interpelle la communauté actuelle des modélisateurs et des épistémologues des modèles et leur lance le défi de montrer que les accusations d'idéologie qu'il déployait il y a presque quarante ans ne sont

Auteur correspondant : fvarenne@wanadoo.fr

F. Varenne est également rattaché au GEMAS (CNRS/UMR 8598),

Fondation Maison des Sciences de l'Homme à Paris. plus valables pour les formes actuelles de modélisation. On y trouvait déjà notamment une forte critique des approches interdisciplinaires fondées sur la modélisation. Or, à l'heure actuelle, il est plus que jamais question d'approches mixtes, systémiques, interdisciplinaires, cela du fait même de l'évolution des objets (systèmes complexes, anthroposystèmes...), de l'évolution de la demande sociale et des problèmes qu'elle soulève en priorité (coordination des expertises pour la résolution de problèmes concrets, renouvellement corrélatif de la recherche-action...), mais aussi de la mise à disposition d'outils à fonction d'intégration (ordinateurs, langages de multi-modélisation ou métamodélisation, plates-formes informatiques...). Cependant, donc, dès 1969, Badiou semblait condamner 
de tels développements futurs : que doit-on en penser aujourd'hui?

Mon objectif ici est limité et, en quelque sorte, préparatoire. Il est de revenir essentiellement sur les arguments principaux de ce livre en permettant une interrogation sur sa pertinence à l'heure où la pratique de modélisation se diversifie encore et que certains de nos collègues manifestent un nouveau scepticisme - sans doute en partie légitime - à l'égard de ce qui peut apparaître comme une tendance au «tout-modélisation » et à l'interdisciplinarité forcée. Pour ce faire, je reviendrai d'abord succinctement sur le contexte dans lequel ce livre a vu le jour, puis je me pencherai sur les thèses clés qu'il déploie.

\section{Le contexte : la critique de l'idéologie dans les sciences}

En 1967, le philosophe et épistémologue Louis Althusser (1974) avait déjà fermement critiqué l'interdisciplinarité dans les sciences. Dans ce qu'il appelait la «mode de l'interdisciplinarité » (ibid, p. 20), il voyait la présence d'idéologies cherchant à s'asservir une partie de la science au nom de catégories philosophiques implicitement enracinées dans des conceptions du monde inavouées : étaient visées les conceptions " positivistes, néo-positivistes, structuralistes, formalistes, phénoménologiques, etc. » (ibid, p. 38) qui toutes étaient accusées de tendre à l'idéalisme. Cependant, les disciplines scientifiques mixtes comme la physique mathématique, la chimie physique, la biophysique et la biochimie n'étaient pas condamnées. Car, selon Althusser, elles manifestaient en elles la présence de rapports organiques de constitution réciproque et non de simple application. Il ne s'agissait pas de cas d'échanges « interdisciplinaires » à proprement parler.

C'est en 1968 que la compétence plus proprement mathématique d'Alain Badiou est mobilisée pour cette lutte contre l'idéalisme qu'Althusser conçoit comme une intervention nécessaire dans la science. Il ne s'agit pas simplement de redire après Lénine que les épistémologies des modèles sont des formations idéologiques réitérées. Mais il s'agit pour Badiou de montrer très précisément le processus même de cette idéologisation de ce qui par ailleurs est devenu un véritable concept des mathématiques, véritablement opérant et à ce titre déjà indiscutable : le concept de modèle.

En 1969, le but de Badiou est donc de détailler le parcours d'une idéologisation, en l'espèce l'idéologisation $\mathrm{d}^{\prime}$ un concept scientifique. Badiou fait fond sur l'idée althussérienne selon laquelle toute idéologie est idéologie pour une science et donc que la lutte contre toute idéologie concerne bien au premier chef l'épistémologie et son devoir d'intervention dans les sciences (les sciences étant des «pratiques » dont les «moyens de production » sont les concepts). Pour Althusser, «la fonction de l'idéologie est alors d'intriquer l'imaginaire et le réel dans une apparence de nécessité pour masquer l'arbitraire [du point de vue de l'individu] des tâches prescrites par le tout social » (Le Bris, 1968, p. 15). La théorie marxiste elle-même reste une pratique du tout social en tant qu'elle travaille sur des concepts donnés par d'autres pratiques. À ce titre, elle s'affronte à l'idéologie et tend en dernière instance à se substituer à elle comme véritable idéologie. Concernant les modèles, Althusser affirmait ainsi que «la conception empiriste du modèle comme idéologie de la connaissance reçoit de la confusion entre l'instrument technique qu'est effectivement un modèle, et le concept de la connaissance, toutes les apparences nécessaires à son imposture » (Althusser, 1968, p. 44). L'imposture de la diffusion de la notion de modèle doit donc être dénoncée.

\section{La critique de l'empirisme logique}

Au début de son livre, Badiou nous révèle d'où vient historiquement cette diffusion indue. Dans le premier chapitre, il rend compte des prétendues «théories de la connaissance » anglo-saxonnes. Il montre qu'on doit d'entrée de jeu rejeter dos à dos leur empirisme et leur formalisme. Pour lui, Carnap (1938) et Quine (1953) sont victimes d'une « formation idéologique particulière » qui se représente la science sous la forme d'une «image » (alors même qu'elle est au contraire un procès et un ensemble de pratiques) ou sous la forme d'une production dont l'objectif est soit la figuration d'objets (empirisme) soit la figuration de formalismes (formalisme). Or, c'est la marque de «l'épistémologie bourgeoise » de se figurer la science à la fois comme figurable et comme productrice de figurations, de représentations en ce sens, puis de se jouer la comédie du balayage apparemment intégral des positions et des variantes possibles.

Badiou entreprend de montrer que Quine (ibid.) - et avec lui toute forme d'alternative à Carnap (ibid.) - n'est que l'envers infiniment différencié de Carnap. En réalité, même si je ne peux le montrer ici, Badiou ne peut faire cette démonstration qu'en occultant les travaux antérieurs de Ernst Nagel (1960), Max Black (1962), Mary Hesse (1966) ou Peter Achinstein (1968) qui, loin de se réduire à l'approche globale et théorique de Quine, avaient tous souligné - et montré par d'innombrables exemples - le rôle éventuellement abusif des modèles en même temps que la grande variété de leurs fonctions épistémiques. Je ne peux que renvoyer ici à la recension critique qu'en fit Pierre Thuillier (1969) dans la revue Atomes et qui n'a aujourd'hui rien perdu de son actualité.

De cette généralisation, Badiou tire en tout cas une thèse forte : «les sciences forment un système discret de différences articulées » tandis que « les idéologies » forment « une combinaison continue de variations» (p. 48). La condamnation de l'interdisciplinarité en est une conséquence inévitable : elle ne peut être qu'une invention 
d'épistémologue qui insère de l'idéologique néfaste dans du scientifique.

Le vrai problème des idéologies infiniment recommencées est donc leur tendance à «imager », à faire devenir " artifice », idole ou image figée, à prétention totalisante mais en réalité sidérante et leurrante, le modèle de ce dont on devrait au contraire respecter la nature dynamique, processuelle et au fond effectivement in-visible, in-figurable et inassignable.

\section{Les trois thèses centrales}

De cette critique préliminaire, Badiou entend tirer trois thèses.

- Thèse 1. Le terme de "modèle » a deux instances épistémologiques : 1) c'est une notion descriptive de l'activité scientifique; 2) c'est un concept de la logique mathématique. Or une notion est une unité du discours idéologique alors qu'un concept est une unité du discours scientifique. Quant à la catégorie, c'est une unité du discours philosophique.

- Thèse 2. Quand l'instance 2) sert de support à 1), on a un recouvrement idéologique de la science, c'est-à-dire une catégorie philosophique : celle de modèle. Reste à savoir si ce recouvrement idéologique favorise un rapport bénéfique avec le concept scientifique de modèle, ou bien, au contraire, si l'épistémologie bourgeoise de la science comme représentation et production de représentations se fige irrémédiablement dans cette pratique idéologique qu'autorise la catégorie philosophique.

- Thèse 3. «La tâche de la philosophie » est de distinguer un usage asservi (aliéné) et un usage "positif » qui se reconnaît à ce qu'il est investi dans LA théorie de l'histoire des sciences (la vraie : le matérialisme historique). Un usage de la catégorie est positif si le modèle y est comme un « adjuvant transitoire destiné à son propre démantèlement». On a un « usage purement idéologique du mot modèle » (chap. 4) en revanche quand on affirme - comme Lévi-Strauss (1958) - que la science est une « connaissance par modèles ». Ainsi, " pour l'épistémologie des modèles, la science n'est pas un procès de transformation pratique du réel, mais la fabrication d'une image plausible». Place est faite à l'idéologie car : « image portative, le modèle unifie extérieurement une politique économique, la légitime et occulte sa cause comme sa règle » (p. 57).

D'où le lien avec le stade du miroir de Lacan (1949) où le réel se fait image et se croit déjà pris en vue surpris - dans l'imaginaire et semble miraculeusement incarné dans le miroir : "Comme l'enfant en vient à surmonter, dans la duperie du miroir, l'horreur de son corps morcelé, les modèles réfléchissent selon l'idéal prématuré du texte unifiant le désordre instantané de la production des savoirs » (p. 55).
C'est ce pouvoir d'unification à la fois factice et prématurée qui est condamné. Plus exactement, c'est la prétention que le modèle peut acquérir par là de se suffire à lui-même sans indiquer sa propre impermanence et son insuffisance.

\section{Conséquences pour les modèles dans les sciences empiriques}

Armé de ces analyses, Badiou montre (chap. 5) qu'il faut refuser de voir les modèles dans les sciences empiriques comme s'inscrivant dans un rapport au réel qui serait parallèle au rapport syntaxe/sémantique tel qu'il existe en théorie mathématique des modèles. En mathématiques, un domaine d'interprétation pour un système formel défini syntaxiquement en est un modèle si à tout énoncé dérivable de ce système formel correspond un énoncé vrai dans ce domaine d'interprétation. Sans s'en apercevoir, l'empirisme logique part d'un rapport intérieur, conceptuel, entre des pratiques qui sont propres à une science (les mathématiques), pour en faire la figuration d'un rapport extérieur entre les sciences et le réel. Mais la distinction syntaxe/sémantique fonctionne conceptuellement en théorie logique ou mathématique des modèles alors qu'elle fonctionne seulement notionnellement en épistémologie des modèles. Là est précisément le lieu d'insertion de l'idéologique dans l'épistémologique : à savoir dans cet usage fallacieux d'une pratique expérimentale et conceptuelle monodisciplinaire ferme et établie pour se figurer la pratique des modèles dans les sciences non purement formelles.

Tout le travail du texte de Badiou va à partir de là consister à tâcher de bien nous rendre claire la nature interne et conceptuelle de ce rapport entre syntaxe et sémantique dans le domaine de la théorie mathématique des modèles. Ce que ne perçoivent pas les idéologues tenant des épistémologies des modèles, c'est «que les critères de la syntaxe pertinente relativement à un modèle donné ne sont pas laissés à l'arbitraire des ressemblances ", et que «ce sont des propriétés théoriques» (p.78) décidées en interne.

C'est là que le sous-titre de l'ouvrage peut se comprendre - et bien sûr en grande partie aussi la préférence originelle de Badiou pour les mathématiques dont il ne se départira pas : «Introduction à une épistémologie matérialiste des mathématiques ». Cette volonté de monstration ou d'imitation des gestes mathématiques en acte est cohérente : puisqu'on n'a plus désormais le droit d'imiter que dans et par l'agir et non dans la figuration - c'est d'ailleurs bien ce qui se révèle finalement comme l'idée fondamentale et commune des anti-idéologismes, anti-idéalismes ou de toutes formes associées d'iconoclasmes (ainsi même des pragmatismes) - la conséquence qu'en tire Badiou et qu'en tirera aussi Desanti, mais aussi donc Althusser et 
ceux qui les suivront à l'époque, est évidemment qu'on ne peut se convaincre de cette internalité et de cette conceptualité à l'œuvre qu'en pratiquant soi-même en interne les mathématiques, c'est-à-dire au fond en faisant d'un livre d'épistémologie quelque chose de très proche d'un livre de mathématiques. Relisant lui-même ce passage, Badiou précise à son propos dans la préface de 2007 : «la didactique est orientée vers une sorte de propagande pour l'écriture formelle, tenue pour la scène où se joue la vérité des concepts » (p. 34).

\section{Modèle et matérialisme en mathématiques : extériorité construite et extériorité plaquée}

Le dernier moment important de l'argumentation de Badiou consiste ainsi à montrer en quoi on a affaire, avec l'élaboration d'un système formel pour un modèle, à une authentique pratique expérimentale, une pratique expérimentale de vérification; ce qui, au passage, lui permet de confirmer de ce point de vue l'historicité tout à la fois dialectique et matérialiste des mathématiques.

Badiou utilise dans ce cadre la célèbre formule de Bachelard selon laquelle les instruments scientifiques sont des «théories matérialisées » (p. 125). Mais par un subtil glissement, il l'acclimate aux mathématiques. Ce qui revient à dire que les systèmes formels axiomatiques sont, comme les instruments de la physique, des théories mathématiques matérialisées. Ce faisant, il semble s'accommoder fort bien d'un renversement du rapport formel/matériel en un rapport matériel/formel assez semblable à celui dont il avait préalablement accusé les empiristes logiques de s'y livrer subrepticement.

Comme ce fut le cas pour le signifiant tel que conçu par Lacan (même si Badiou se départira de cette référence directe par la suite, cf. p. 26-27), c'est dans l'écriture mathématique, à savoir dans la matérialité de ces inscriptions symboliques, que Badiou affirme dans un deuxième temps reconnaître la matérialité des marques de l'instrument expérimental propre aux mathématiques. Pour Badiou, en dernière analyse, c'est cette matérialité de l'écriture réglée du système formel qui la constitue de fait en instruments, c'est-à-dire la fait entrer dans la catégorie d'instrument : les machines déductives matérielles que sont les ordinateurs en sont l'illustration patente. Une question demeure pour le lecteur : cette catégorie transdisciplinaire $\mathrm{d}^{\prime}$ « instrument matériel » ne devient-elle pas à son tour le symptôme d'un plaquage et d'une extension indue, donc une simple notion développant la mauvaise idéologie?

À juste titre cependant, Badiou invoque ici la condition de calculabilité effective qui fait selon lui écho au seul principe d'identité qui vaille (en physique) selon Bachelard : «le principe d'identité des instruments ». C'est donc précisément là qu'il y a un rapport interne, monodisciplinaire, qui semble pouvoir passer illusoirement pour un rapport à un dehors des mathématiques, un rapport interdisciplinaire, qui vaudrait comme un rapport de représentation ou de figuration du tout autre (à savoir du réel) dans ou à partir des mathématiques, c'est-à-dire en en partant, en en sortant.

Il faut comprendre au contraire que le rapport syntaxe/sémantique en mathématique institue la sémantique comme « un rapport intra-mathématique entre certains dispositifs expérimentaux raffinés (les systèmes formels) et certains produits mathématiques plus "grossiers", c'està-dire acceptés, tenus pour démontrés, sans avoir été soumis à toutes les exigences d'inscription dont le dispositif règle la contrainte vérifiante » (p. 124).

Le procédé d'idéologisation qui est le cœur du propos de Badiou prend exactement son origine ici : dans la confusion entre une extériorité construite de l'intérieur d'une discipline scientifique pour les besoins du jeu praxique qui doit lui être propre, c'est-à-dire de ce jeu d'une histoire dialectique propre (chap. 10) à ce qui se présente d'abord comme une pratique sociale et matérielle (notamment d'écriture) et un rapport d'extériorité plat, extérieurement imposé. C'est une confusion entre un rapport d'extériorité construit de l'intérieur d'une théorie effectivement pratiquée et un rapport d'extériorité extérieurement imposé, car en partie déterminé par des intérêts de classe. Ce rapport extérieurement imposé est idéologiquement déterminé car déterminé artificieusement à partir de figurations naïves de la science puis du réel, figurations non théoriquement déterminées ni communément mises en pratique ou soumises à pratiques dans le cœur d'une activité théorique contrôlée par des instruments à la matérialité avérée et vérifiante : "C'est parce qu'il est lui-même théorie matérialisée, résultat mathématique, que le dispositif formel peut entrer dans le procès de production des connaissances mathématiques ; et dans ce procès, le concept de modèle ne désigne pas un dehors à formaliser, mais un matériau mathématique à éprouver » (p. 133).

Pour le Badiou de 1969, aucune autre articulation (qui serait donc interdisciplinaire) n'est possible. Car noblesse native oblige : le matériau à quoi s'applique l'instrument formel doit être de noble extraction. Il doit être de la nature de ce préalable mathématique ${ }^{1}$, de ce qui possède toujours la vocation de devenir modèle, de ce qui est toujours déjà appelé par l'aventure dialectique du savoir à devenir modèle. C'est sur ce chemin de croix, sur le chemin de l'épreuve, entendue comme mise en pratique et matérialisation, que les modèles gagnent leur droit à être nommés ainsi.

\footnotetext{
${ }^{1}$ C'est le sens du «mathématisme » ou «platonisme » qu'il revendique encore en 2007 (pp. 21 et 24). En 1969, il écrivait : "parler de modèles, c'est présupposer la "vérité" (l'existence) de ces pratiques mathématiques [sc. théorie des ensembles, axiome d'induction...]. On s'établit dès le début dans la science. On ne la reconstitue pas à partir de rien. On ne la fonde pas » (p. 104).
} 


\section{Bilan sur les modèles : adjuvants transitoires ou productions symboliques consacrées}

Au final donc, les modèles sont soit des adjuvants transitoires qui doivent être niés dialectiquement pour que le matérialisme historique se confirme dans l'histoire même des sciences, soit des matériaux mathématiques encore informes ou démontrés mais non prouvés et qui prennent corps - s'incarnent - par l'effet d'une instrumentation (axiomatique) inséparable d'une pratique réalisante et consacrante. Cette consécration qui est en même temps rétrospection et rétroaction est possible, car elle bénéficie des forces symboliques de production par quoi le signifiant du dispositif formel peut forcer le matériau mathématique informe à se produire dans une expérimentation décisive et donc obtient le droit réel de le consacrer. Il n'y aurait plus ici d'idéologique car il n'y aurait nulle intervention du réel ou de l'imaginaire, tout au moins comme figuration.

Comme dans tous les iconoclasmes épistémologiques, $c^{\prime}$ est ici la pratique qui finit par être consacrante, puis consacrée (par l'épistémologue). Mais on peut dire que c'est une pratique fermée et exclusive. Ce n'est pas celle des pragmatismes anglo-saxons : pour Badiou, la pratique sécrète sa propre production et consacre ensuite rétroactivement ses propres produits pour créer des frontières - en toute liberté - mais à l'intérieur d'elle-même. Ce modèle de la liberté mathématique auquel la préface de 2007 rend encore hommage est celui d'une liberté très surveillée, résolument cartésienne en ce sens (pp. 20 et 22).

Comme la conception sémantique qu'elle entreprend de critiquer, cette approche unitaire des modèles pose de considérables problèmes que je n'ai pu qu'évoquer, à commencer par cette consécration exclusive des mathématiques (voire d'une certaine interprétation des mathématiques) et la dénonciation radicale de l'interdisciplinarité. De fait, de 1969 à nos jours, ce livre sera invoqué surtout pour fustiger les dérives des usages représentationnalistes des modèles, mais rarement pour critiquer le développement même des modèles formels dans les sciences. À côté de sa place incontestable dans l'histoire de l'épistémologie française des modèles, cet ouvrage garde toute sa force et tout son pouvoir d'attraction, mais surtout du point de vue d'une épistémologie des mathématiques.

\section{Conclusion : à quoi Badiou a-t-il dit non?}

Sans émettre de critiques plus avancées sur cet ouvrage ici (ce n'est pas mon objectif), je formulerai pour finir une suggestion qui prend la forme d'une simple prolongation de l'argumentaire de l'auteur : ce "principe d'identité des instruments » appliqué à nos usages contemporains des ordinateurs (et non uniquement à celui - purement déductif - auquel pense encore Badiou, et, en 1969, c'est légitime) ne peut-il servir aujourd'hui à élargir la notion de «théories matérialisées » dans l'instrument et ainsi, finalement, à atténuer la condamnation de l'interdisciplinarité ? En effet, les allers-retours modélisation-simulation en régime interdisciplinaire ne sont-ils pas devenus - à l'image de la pratique d'axiomatisation formelle en mathématiques - une forme de pratique inséparablement matérielle et formelle de vérification pour ce qui ne serait effectivement sinon qu'une concaténation d'énoncés formant un «corps d'énoncés » prématuré (p. 55), faussement « incarné » (donc une idole) et, de ce fait, idéologique?

À mon avis, la fortune actuelle des divers pragmatismes anglo-saxons pour la pensée des modèles au détriment du «praxisme » continental d'origine marxiste des années 1960 trouverait là une de ses explications. Ces pragmatismes anglo-saxons aujourd'hui dominants (et contre lesquels Badiou veut nouvellement combattre) sont d'origine certes empiriste, mais surtout pluraliste, comme de jeunes chercheurs nous l'ont récemment découvert ou redécouvert ( $c f$. la récente présentation par Stéphane Madelrieux, dans une nouvelle traduction, de Le pragmatisme de James (1907)). Comme naguère les praxismes marxistes le firent, ces pragmatismes rendent aujourd'hui l'épistémologie des modèles toujours plus sensibles à l'action, à la pratique, mais, cette fois-ci, sans l'enfermer dans une théorie monolithique de la pratique, théorie monolithique ici car surtout spéculative et, on l'a vu, issue d'une seule science décrétée reine (les mathématiques).

Comme on peut s'en apercevoir, l'histoire de l'épistémologie des modèles est aussi l'histoire de leurs critiques. Or, pas plus qu'elle n'est séparable de l'histoire des sciences et des techniques, elle n'est séparable de l'histoire de la philosophie elle-même, de ses récurrences, et, éventuellement, de la récurrence des discours politiques et anthropologiques associés. En ce sens, dire non au pluralisme tout en enjoignant les uns et les autres - toutes disciplines confondues - à sacrifier au culte de l'action, y compris dans sa dimension sociale, est bien la teneur d'un discours qui a défini un certain moment de la pensée française.

\section{Références}

Achinstein, P., 1968. Concepts of Science - A philosophical analysis, Baltimore and London, The Johns Hopkins Press.

Althusser, L., 1974. Philosophie et philosophie spontanée des savants (1967), Paris, Maspero, série de cours donnés à l'ENS en 1967.

Althusser, L., 1968. Lire le Capital, Tome 1, Paris, Maspéro.

Badiou, A., 1969. Le concept de modèle - Introduction à une épistémologie matérialiste des mathématiques, Paris, Maspero, collection Théorie dirigée par Althusser, L. ; réimpression avec une nouvelle préface de l'auteur : Paris, Fayard, 2007.

Black, M., 1962. Models and Metaphors - Studies in Language and Philosophy, Ithaca and London, Cornell University Press. 
Carnap, R., 1938. Logical Foundations of the Unity of Science in Foundations of the Unity of Science. Toward an International Encyclopedia of United Science, vol. I, 1-10, Neurath, O., Carnap, R., Morris, C. (Eds), The University of Chicago Press, Cloth Edition published in 1955, $3^{\text {rd }}$ impression : 1971, $42-75$.

Hesse, M. B., 1966, Models and Analogies in Science, Notre Dame, University of Notre Dame Press.

James, W., 1907, Le pragmatisme; nouvelle traduction par Ferron N. ; présentation par Madelrieux S., Paris ChampsFlammarion, 2007.

Lacan, J., 1949. Le stade du miroir comme formateur de la fonction du Je telle qu'elle nous est révélée dans l'expérience psychanalytique, repris in Ecrits I, Paris, Seuil, 1999, 92-99.
Le Bris, M., 1968. Althusser, une nouvelle analyse de Marx, Le magazine littéraire, avril, 14-15.

Lévi-Strauss, C., 1958, Anthropologie structurale, Paris, Plon.

Nagel, E., 1960, The Structure of Science, Indianapolis, Hackett Publishing Company.

Quine, W.V.O., 1953, From a Logical Point of View, Harvard, Cambridge MA ; trad. : D'un point de vue logique, Paris, Vrin, 2003.

Thuillier, P., 1969. L'épistémologie des modèles est-elle bourgeoise?, Atomes, n² 269, 626-631. 